\title{
University of Missouri-St. Louis
}

From the SelectedWorks of Bruce Wilking

January 3, 1990

\section{The Circumstellar Structure of IRAS 16293-2422: $\mathrm{C} 18 \mathrm{O}, \mathrm{NH} 3$, and CO Observations}

Lee G. Mundy, University of Maryland, College Park

H. A. Wootten

Bruce A. Wilking, National Radio Astronomy Observatory 
THE ASTROPHYSICAL JOURNAL, 352:159-166, 1990 March 20

(C) 1990. The American Astronomical Society. All rights reserved. Printed in U.S.A.

\title{
THE CIRCUMSTELLAR STRUCTURE OF IRAS 16293-2422: $\mathrm{C}^{18} \mathrm{O}, \mathrm{NH}_{3}$, AND CO OBSERVATIONS
}

\author{
Lee G. Mundy ${ }^{1}$, H. A. Wootten ${ }^{2}$, AND Bruce A. Wilking ${ }^{3}$ \\ Received 1989 June 23 ; accepted 1989 September 19
}

\begin{abstract}
Interferometric observations of the $\mathrm{C}^{18} \mathrm{O}$ and ${ }^{12} \mathrm{CO} J=1-0$ transitions, and the $\mathrm{NH}_{3}(1,1)$ and $(2,2)$ transitions toward the young far-infrared source IRAS 16293-2422 are presented. The $\mathrm{C}^{18} \mathrm{O}$ and $\mathrm{NH}_{3}$ data reveal elongated gas structures which are aligned perpendicular to the axis of a bipolar outflow and to the local magnetic field direction. Assuming a distance of $160 \mathrm{pc}$, the $\mathrm{C}^{18} \mathrm{O}$ emission arises from a $2000 \mathrm{AU}$ diameter region which is coextensive with $40 \mathrm{~K}$ dust emission and shows a velocity shift of $2.4 \mathrm{~km} \mathrm{~s}^{-1}$ consistent with rotational motion about an included mass of 1.5-1.7 $M_{\odot}$. The $\mathrm{NH}_{3}$ emission comes from an $8000 \mathrm{AU}$ diameter region with a kinetic temperature of $15-20 \mathrm{~K}$ and a total mass of $0.2 M_{\odot}$. Interferometric $\mathrm{CO}$ $J=1-0$ observations show that IRAS 16293-2422 is also responsible for at least part of the large scale CO outflow seen in the area.

By combining the interferometric data with single-dish spectra of the $\mathrm{C}^{18} \mathrm{O}$ and $\mathrm{C}^{17} \mathrm{O}$ lines, a comprehensive model for the IRAS 16293-2422 region is developed. In this model, the compact $\mathrm{C}^{18} \mathrm{O}$ line and $40 \mathrm{~K}$ dust emission arise from rotating material within which the central heat source(s) is embedded. $\mathrm{The}^{\mathrm{NH}} \mathrm{NH}_{3}$ emission is distributed in a ring with radius 3000 to 4000 AU surrounding the central disk. The dearth of ammonia emission from the $\mathrm{C}^{18} \mathrm{O}$ emission region is attributed to an actual decrease in $\mathrm{NH}_{3}$ abundance.
\end{abstract}

Subject headings: infrared: sources - interstellar: molecules - nebulae: individual (IRAS 16293-2422)

\section{INTRODUCTION}

Since its initial identification as a low-luminosity $\left(27 L_{\odot}\right)$ protostellar candidate (Walker et al. 1986), IRAS $16293-2422$ has been the subject of numerous high-resolution millimeter and centimeter wavelength observations which have revealed considerable structure within this nearby $(160 \mathrm{pc})$ source. The simple spherically symmetric infall model of Walker et al. (1986) has been challenged by maps of a more extended absorbing layer of gas (Menten et al. 1987). Mundy, Wilking, and Myers (1986) found the infrared and millimeter continuum emission to arise from an 1800 by $<800$ AU high column density structure within the proposed infall region. A group of water masers (Wilking and Claussen 1987; Wootten 1989a) and two regions of centimeter radio emission separated by 750 AU (Wootten 1989a) have since been discovered within the dust emission region, indicating the presence of outflow activity in the heart of the source.

In addition to this intriguing complexity, IRAS $16293-2422$ is the nearest example of a deeply embedded young stellar object which radiates nearly $100 \%$ of its luminosity in the far-infrared spectral region. It is unique among low-luminosity objects in the strength of its millimeter wavelength continuum flux and associated $\mathrm{H}_{2} \mathrm{O}$ maser emission. This combination of excellent linear resolution and strong emission makes IRAS 16293-2422 an ideal source for detailed studies of the circumstellar environment and gas dynamics during the earliest stages of stellar formation.

This paper contains new interferometric observations of the $\mathrm{CO} J=1-0, \mathrm{C}^{18} \mathrm{O} J=1-0, \mathrm{NH}_{3}(1,1)$, and $\mathrm{NH}_{3}(2,2)$ emissions from the IRAS 16293-2422 region with a spatial resolution of $800-1600 \mathrm{AU}$. These data confirm the previous suggestion of rotation within the millimeter emission region

\footnotetext{
${ }^{1}$ Owens Valley Radio Observatory, California Institute of Technology.

2 National Radio Astronomy Observatory.

${ }^{3}$ Physics Department, University of Missouri-St. Louis.
}

(Mundy, Wilking, and Myers 1986; Menten et al. 1987) and reveal the presence of a larger scale disk-like structure extending out to a radius of $4000 \mathrm{AU}$.

\section{OBSERVATIONS}

Interferometric and single-dish spectral line observations were obtained toward IRAS 16293-2422 using a pointing center of $\alpha_{1950}=16^{\mathrm{b}} 29^{\mathrm{m}} 20^{\mathrm{s}} 9, \delta_{1950}=-24^{\circ} 22^{\prime} 13^{\prime \prime}$ and central velocity of $4.0 \mathrm{~km} \mathrm{~s}^{-1}$. These observations are described below and summarized in Table 1.

\section{a) Interferometric $\mathrm{C}^{18} \mathrm{O}$ and ${ }^{12} \mathrm{CO}$ Observations}

The $J=1-0$ transitions of $\mathrm{C}^{18} \mathrm{O}$ and ${ }^{12} \mathrm{CO}$ (hereafter $\mathrm{CO}$ ) were observed using the Owens Valley Radio Observatory (OVRO) 3 element Millimeter Wave Interferometer. Data from 32 channels with $1 \mathrm{MHz}\left(2.6 \mathrm{~km} \mathrm{~s}^{-1}\right)$ resolution and 32 channels with $50 \mathrm{kHz}\left(0.13 \mathrm{~km} \mathrm{~s}^{-1}\right)$ resolution were obtained simultaneously with a $350 \mathrm{MHz}$ wide continuum channel. The $\mathrm{CO}$ data were taken toward two pointing centers spaced 21 " east and west of the IRAS $16293-2422$ position. The point source $1730-130$ was observed as a phase calibrator. The data were amplitude calibrated based on Mars $\left(T_{B}=207 \mathrm{~K}\right)$ and $3 \mathrm{C} 84$ ( $30 \mathrm{Jy}$ ). The absolute positional uncertainty is estimated to be 0.2 of the synthesized beam, $1^{\prime \prime} 0$ for the $\mathrm{C}^{18} \mathrm{O}$ data and $1 . .5$ for the CO data. The absolute flux uncertainty is estimated to be $20 \%$.

\section{b) Single-Dish Isotopic $\mathrm{CO}$ Observations}

Observations of $\mathrm{C}^{18} \mathrm{O}$ and $\mathrm{C}^{17} \mathrm{O}$ were obtained using the NRAO $^{4} 12 \mathrm{~m}$ for the $J=1-0$ transitions and the University of Texas Millimeter Wave Observatory (MWO) ${ }^{5} 4.9 \mathrm{~m}$ for the

\footnotetext{
${ }^{4}$ The National Radio Astronomy Observatory is operated by Associated Universities, Inc., under cooperative agreement with the National Science Foundation.

${ }^{5}$ The Millimeter Wave Observatory is operated by the Electrical Engineering Research Laboratory, the University of Texas at Austin, with support from the National Science Foundation and McDonald Observatory.
} 
TABLE 1

OBSERVATIONS

\begin{tabular}{|c|c|c|c|c|c|c|}
\hline Transition & Observatory & Date & $\begin{array}{c}\text { Primary Beam } \\
\text { (arcsec) }\end{array}$ & $\begin{array}{l}\text { Synthesized Beam } \\
\text { (arcsec) }\end{array}$ & $\begin{array}{c}\Delta V^{\mathrm{a}} \\
\left(\mathrm{km} \mathrm{s} \mathrm{s}^{-1}\right)\end{array}$ & $\begin{array}{c}\text { Bandwidth }^{\mathrm{a}} \\
\left(\mathrm{km} \mathrm{s}^{-1}\right)\end{array}$ \\
\hline $\mathrm{NH}_{3}(1,1)$ & VLA & $1987 \mathrm{Feb}$ & 120 & $6.0 \times 6.0^{b}$ & 0.61 & 19.8 \\
\hline $\mathrm{NH}_{3}(2,2) \ldots \ldots \ldots \ldots \ldots \ldots$ & VLA & 1988 May & 120 & $13 \times 13^{\mathrm{c}}$ & 0.61 & 19.8 \\
\hline $2.7 \mathrm{~mm}$ continuum $\ldots \ldots \ldots$ & OVRO & 1986 Nov-1987 Feb & 68 & $6.3 \times 4.5$ & $\ldots$ & $350 \mathrm{MHz}$ \\
\hline $\mathrm{C}^{18} \mathrm{O} J=1-0 \ldots \ldots \ldots \ldots$ & OVRO & 1986 Nov-1987 Feb & 68 & $6.3 \times 4.5$ & $0.27,2.73$ & $7.1,90.1$ \\
\hline $\mathrm{C}^{18} \mathrm{O} J=1-0 \ldots \ldots \ldots \ldots$ & NRAO & 1985 Jun & 65 & $\ldots$ & 0.27 & 34.6 \\
\hline $\mathrm{C}^{18} \mathrm{O} J=2-1 \ldots \ldots \ldots \ldots$ & MWO & $1987 \mathrm{Apr}$ & 70 & $\ldots$ & $0.085,0.34$ & $10.9,87.0$ \\
\hline $\mathrm{C}^{17} \mathrm{OJ}=1-0 \ldots \ldots \ldots \ldots$ & NRAO & $1987 \mathrm{Apr}$ & 65 & $\ldots$ & $0.080,0.27$ & $10.2,34.6$ \\
\hline $\mathrm{C}^{17} \mathrm{O} J=2-1 \ldots \ldots \ldots \ldots$ & MWO & $1987 \mathrm{Apr}$ & 70 & $\ldots$ & $0.083,0.33$ & $10.7,85.4$ \\
\hline $\operatorname{CO} J=1-0 \ldots \ldots \ldots \ldots \ldots$ & OVRO & 1987 Jan-1987 May & 66 & $10.3 \times 5.3$ & $0.26,2.60$ & $4.2,83.2$ \\
\hline
\end{tabular}

a Two velocity resolutions obtained simultaneously.

b Natural weighting with $40 \mathrm{k} \lambda$ taper.

c Natural weighting with $18 \mathrm{k} \lambda$ taper.

$J=2-1$ transitions. The resulting observations of the $J=1-0$ and $J=2-1$ transitions have very similar angular resolution. The $12 \mathrm{~m}$ data were corrected for telescope efficiency assuming $\eta_{\text {FSs }}=0.74$ and $\eta_{\mathrm{C}}=0.9$. The MWO observations were corrected for efficiency assuming $\eta_{\mathrm{FSS}}=0.83$ and $\eta_{\mathrm{C}}=0.75$. The estimated uncertainty in the absolute temperature scale is $20 \%$, mainly due to uncertainty in the source emission distribution and the absolute temperature of Jupiter $(170 \mathrm{~K})$, the primary standard for both sets of observations.

\section{c) $\mathrm{NH}_{3}$ Observations}

Observations in the $\mathrm{NH}_{3}(J, K)=(1,1)$ and $(2,2)$ lines were made in the $C / D$ configuration with the NRAO Very Large Array (VLA). For the $(1,1)$ line observations, 25 antennas were used with a correlator arrangement consisting of $3248 \mathrm{kHz}$ $\left(0.61 \mathrm{~km} \mathrm{~s}^{-1}\right)$ channels centered on the main hyperfine components at $23.694506 \mathrm{GHz}$. The frequency coverage of the correlator also included the inner satellite hyperfine components of the $(1,1)$ line. For the $(2,2)$ line observations, 26 antennas were employed, 17 of which had newly installed HEMT receivers providing improved sensitivity. Data in $3224 \mathrm{kHz}(0.3 \mathrm{~km}$ $\mathrm{s}^{-1}$ ) channels centered on the main hyperfine components at 23.722634 GHz were obtained. The point source $1622-297$ served as the phase calibrator, and 3C 286 (2.52 Jy) was the flux standard. The absolute positional uncertainty is estimated to be 0.1 of a beam: 0 ". 6 for the $6^{\prime \prime}$ tapered beam of the $\mathrm{NH}_{3}(1,1)$ data and 1".3 for the $13^{\prime \prime}$ tapered beam of the $\mathrm{NH}_{3}(2,2)$ data. The absolute flux uncertainty is estimated to be $20 \%$.

\section{d) Reduction Techniques for Interferometer Data}

Maps of the interferometric $\mathrm{C}^{18} \mathrm{O}$ and $\mathrm{CO}$ data were made using the CLEAN algorithm in AIPS with uniform weighting and no taper, resulting in the synthesized beams listed in Table 1. The $50 \mathrm{kHz}$ resolution data were averaged to $100 \mathrm{kHz}(0.27$ $\mathrm{km} \mathrm{s} \mathrm{s}^{-1}$ ) resolution to improve the signal to noise. The CLEANed CO maps from the two pointing centers were corrected for primary beam response and combined by weighted averaging of the maps with weights proportional to the square of the power in the primary beams. The rms noise level in the $\mathrm{C}^{18} \mathrm{O}$ maps with $100 \mathrm{kHz}$ resolution is $0.6 \mathrm{Jy}^{\text {beam }}{ }^{-1}$; the rms noise in the $\mathrm{CO}$ maps with $1 \mathrm{MHz}$ resolution is $0.4 \mathrm{Jy}_{\text {beam }}^{-1}$. The corresponding brightness temperature sensitivities are 2.1 and $0.7 \mathrm{~K}$, respectively.

For the $\mathrm{NH}_{3}(1,1)$ data, both CLEAN and MEM maps were made with a $40 \mathrm{k} \lambda$ taper, sacrificing the full resolution $(\sim 2$ ". 8 ) to improve the brightness temperature sensitivity. The final
CLEAN maps, using a 6" synthesized beam, have an rms noise level of $40 \mathrm{mJy}^{\text {beam }}{ }^{-1}$ or $3.0 \mathrm{~K}$ brightness temperature. Both the CLEAN and MEM maps gave very similar results; only the CLEAN maps convolved with a $6^{\prime \prime}$ beam are presented.

The emission from the $\mathrm{NH}_{3}(2,2)$ line was weaker than that from the $(1,1)$ line and consequently required more spatial averaging to obtain reasonable signal to noise. To determine the optimal resolution for display of the data, untapered maps were made and Fourier transformed. Real and complex images were then added in quadrature to produce images in the Fourier plane. Inspection of these images revealed clear structure on scales smaller than $18 \mathrm{k} \lambda$. New maps were made with that taper to optimally match brightness temperature sensitivity and the scale of structure in the source. The resulting beam size is $13^{\prime \prime}$ and the sensitivity of the CLEAN maps is 33 mJy beam ${ }^{-1}$, corresponding to $0.42 \mathrm{~K}$. Both CLEAN and MEM maps were produced. The latter better represent weak extended structure and are used for image analysis. Spectral analysis employed the CLEAN maps for the channels where signal was present.

\section{RESULTS}

\section{a) $\mathrm{C}^{18} \mathrm{O}$ and Continuum Emission}

Maps of the integrated $\mathrm{C}^{18} \mathrm{O}$ and the $2.72 \mathrm{~mm}$ continuum emission are shown in Figure 1. The continuum map is identical to the previous map by Mundy, Wilking, and Myers (1986) to within the noise in the two data sets. The $\mathrm{C}^{18} \mathrm{O}$ source size, at the $2 \mathrm{Jy} \mathrm{km} \mathrm{s}^{-1}$ beam $^{-1}$ level, is $12^{\prime \prime} .4( \pm 0$ ".4) $\times 7$ ". $3( \pm 0.4)$ with position angle $150^{\circ}\left( \pm 7^{\circ}\right)$ which is nearly identical to the continuum source size at the $100 \mathrm{mJy}^{\text {beam }}{ }^{-1}$ level. Like the continuum emission, the $\mathrm{C}^{18} \mathrm{O}$ emission is unresolved along the minor axis. The centroid of the $\mathrm{C}^{18} \mathrm{O}$ emission is coincident with the centroid of the continuum source to within the $2 \sigma$ errors but the peak of the $\mathrm{C}^{\mathbf{1 8}} \mathrm{O}$ emission is shifted along the minor axis, 2 ". 4 east and 2 ". 1 south, relative to the continuum peak. Since the relative position errors between the continuum and line data (acquired simultaneously) are $0.1-0.15$ of the synthesized beam $(0.5-0$ ". 8$)$, the separation in the two emission peaks is significant at the 3-4 $\sigma$ level.

Figure 2 is a contour map of the velocity field along the major axis (integrated across the minor axis) of the $\mathrm{C}^{18} \mathrm{O}$ emission region. There is a clear shift in velocity with position along the major axis. Distinct emission peaks occur at $V_{\mathrm{LSR}}=5.0$ and $2.9 \mathrm{~km} \mathrm{~s}^{-1}$ with relative positions of $-2^{\prime \prime}$ and $+4^{\prime \prime}$, respectively. The mean velocities of the emission at opposite ends of 


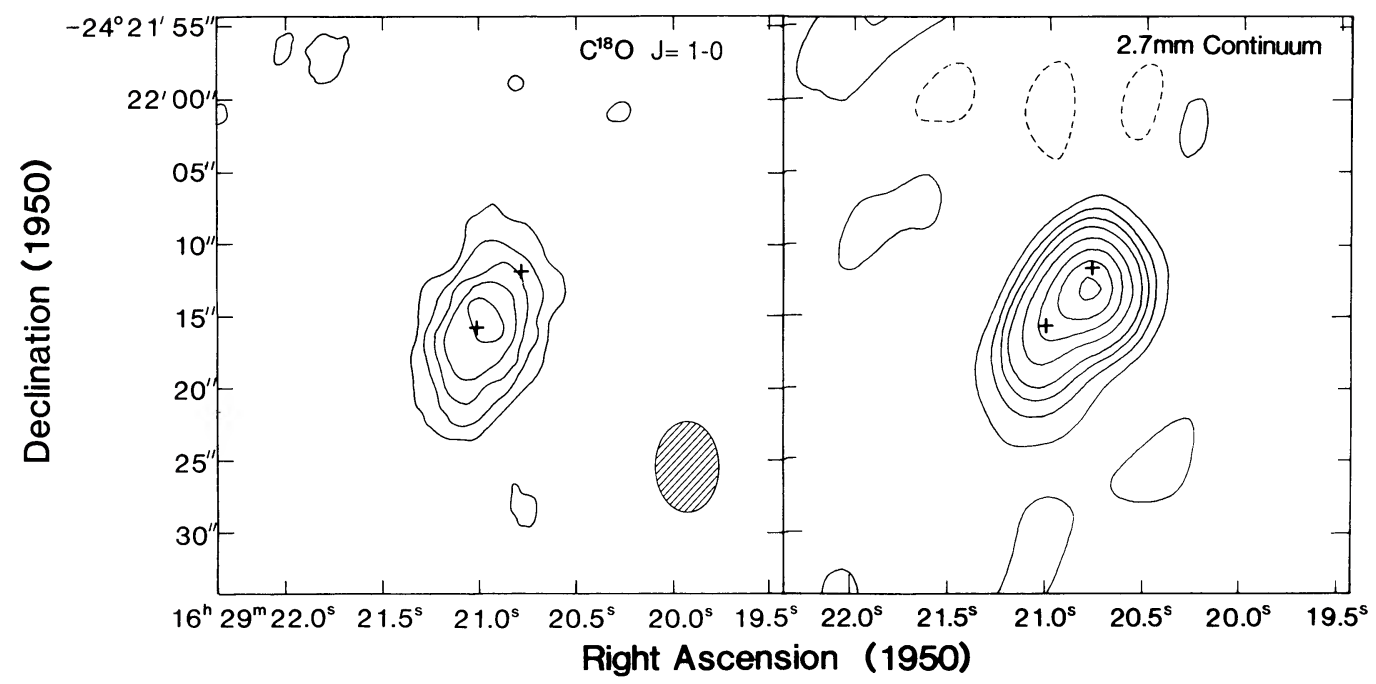

Fig. 1.-Maps are shown of the integrated $\mathrm{C}^{18} \mathrm{O} J=1-0$ (left) and $2.7 \mathrm{~mm}$ continuum emission (right) from IRAS $16293-2422$. The contours in the $\mathrm{C}^{18} \mathrm{O}$ map are $1.0,2.0,3.0,4.0$, and $5.0 \mathrm{Jy} \mathrm{km} \mathrm{s}^{-1}$ beam ${ }^{-1}$. The contours in the continuum map are $-25,25,50,75,100,150,200,250$, and $300 \mathrm{mJy} \mathrm{beam}^{-1}$. The beam for both maps is shown in the lower right corner of left panel. The pluses in both maps mark the positions of the 2 and $6 \mathrm{~cm}$ radio sources (Wootten 1989a).

the major axis, $\pm 6^{\prime \prime}$, are 5.15 and $2.75 \mathrm{~km} \mathrm{~s}^{-1}$, respectively. The peak emission in the individual $100 \mathrm{kHz}$ channel maps is 3-3.8 $\mathrm{Jy}_{\text {beam }}{ }^{-1}$ corresponding to $10-13.5 \mathrm{~K}$ brightness temperature.

Comparison of single-dish spectra with the emission measured by the interferometer observations (Fig. 3) clearly indicates that most of the single-dish flux, peaking at $135 \mathrm{Jy}$ in a $1^{\prime}$ beam, arises on scales $>35^{\prime \prime}$ which were not sampled in the interferometer observations. The interferometer observations show flux across the entire width of the single-dish line with the fraction of recovered flux approaching $50 \%$ in the outer portions of the line. The decreased flux at line center in Figure 2 does not necessarily reflect a true decrease in emission. Gas in the surrounding cloud, which probably contributes the major-

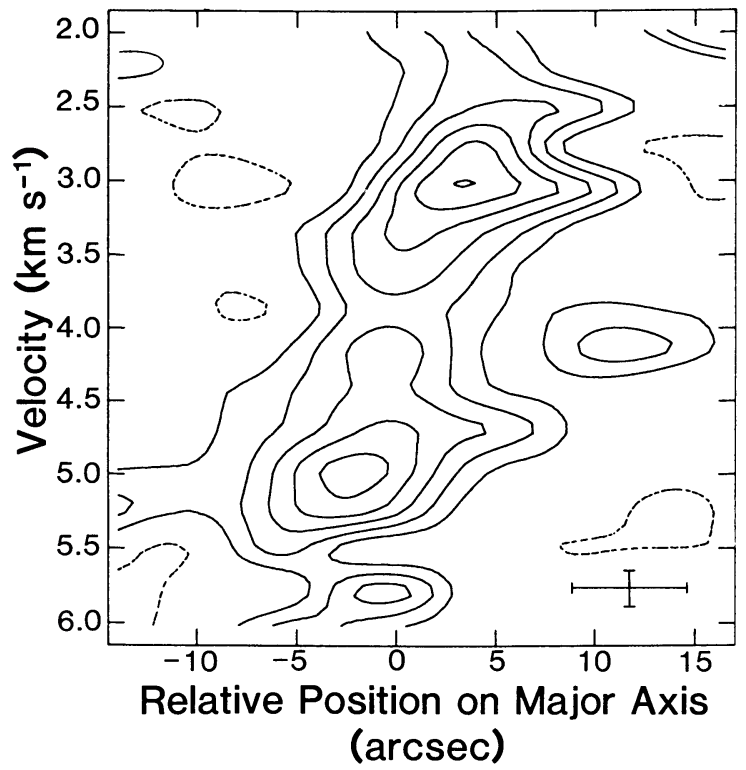

FIG. 2.-Velocity-position map of the $\mathrm{C}^{18} \mathrm{O} J=1-0$ emission along the major axis of the source is plotted. The zero relative position on the major axis is $\alpha_{1950}=16^{\mathrm{h}} 29^{\mathrm{m}} 20^{\mathrm{s}} 9, \delta_{1950}=-24^{\circ} 22^{\prime} 13^{\prime \prime}$. The error bars in the lower right corner indicate the beam size projected on the major axis and the velocity width of a channel. ity of the single-dish flux, may significantly attenuate the emission around $4 \mathrm{~km} \mathrm{~s}^{-1}$ from the immediate vicinity of IRAS $16293-2422$. The nature of the absorbing gas will be discussed further in $\S$ IV.

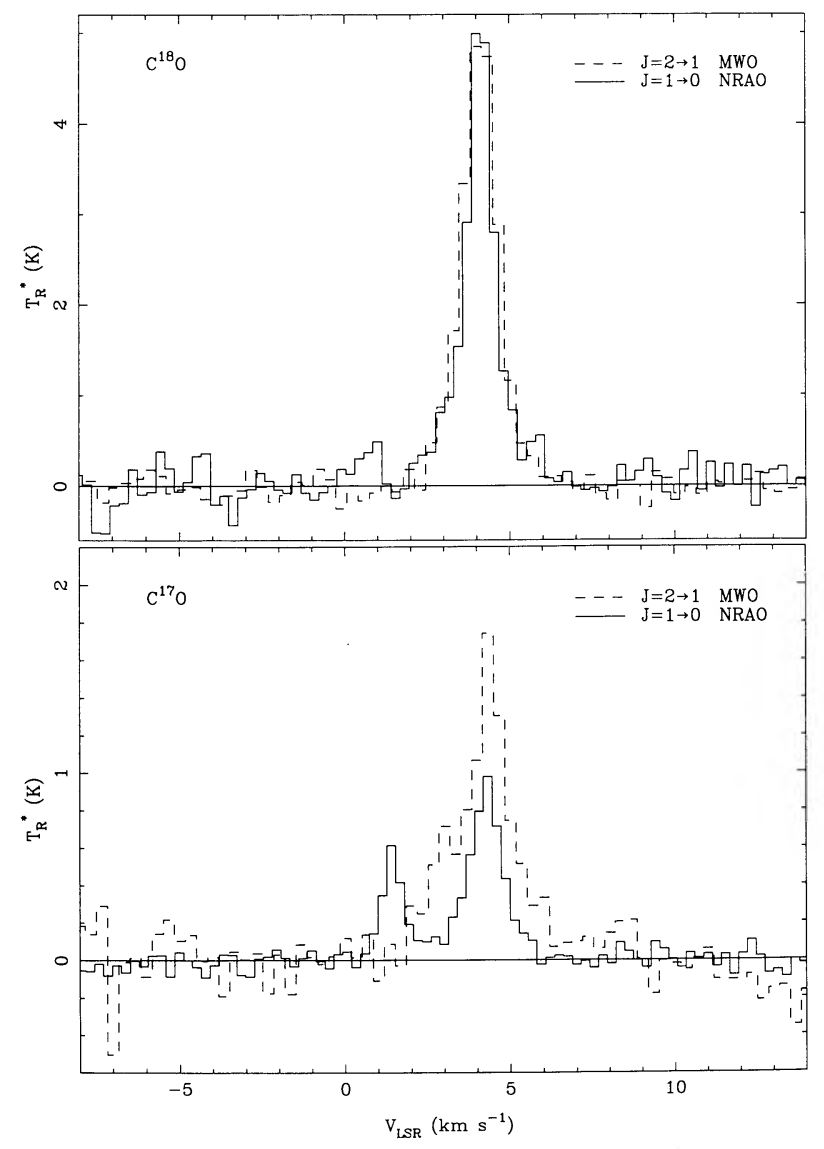

Fig. 3.-Single-dish spectra of the $J=1-0$ and $J=2-1$ transitions of $\mathrm{C}^{18} \mathrm{O}$ and $\mathrm{C}^{17} \mathrm{O}$ are displayed. The $J=1-0$ spectra are from the NRAO $12 \mathrm{~m}$ and the $J=2-1$ spectra are from the MWO $4.9 \mathrm{~m}$. All spectra have been scaled to units of $T_{R}^{*}$ using the telescope efficiencies given in $\S$ II $c$. 


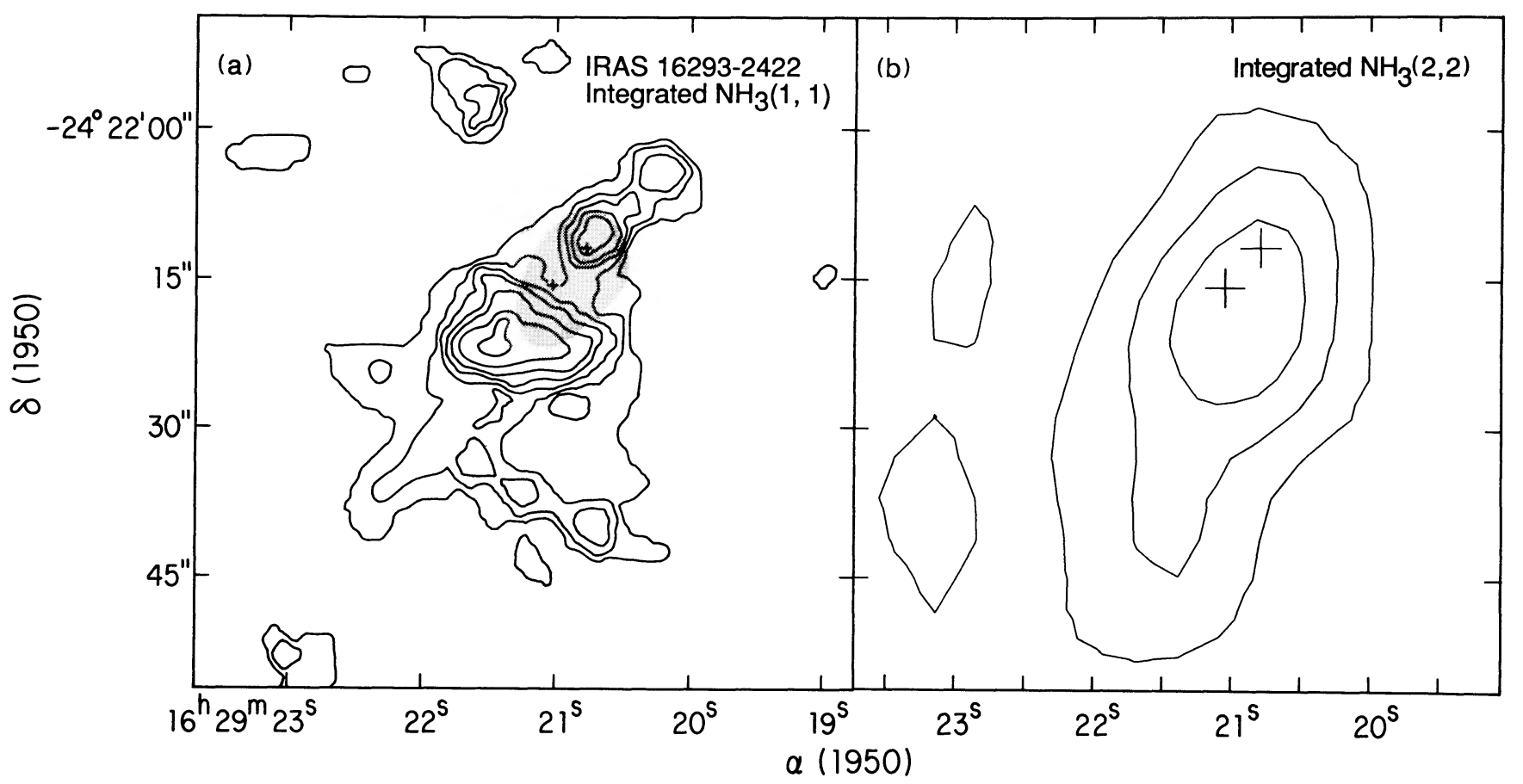

FIG. 4. - (a) A map of the integrated $\mathrm{NH}_{3}(1,1)$ emission from IRAS $16293-2422$ is shown. The contour levels are in steps of $60 \mathrm{mJy}^{\mathrm{beam}} \mathrm{j}^{-1} \mathrm{~km} \mathrm{~s}^{-1}$. The synthesized beam, given in the lower right corner, is $6^{\prime \prime} \times 6^{\prime \prime}$. The $\mathrm{C}^{18} \mathrm{O}$ emission is shown by the stippled region and plus marks denote the positions of the $6 \mathrm{~cm}$ radio sources (Wootten 1989a). (b) A map of the integrated $\mathrm{NH}_{3}(2,2)$ emission is displayed. The contour levels are $.2, .4$, and .8 of the peak flux in the maximum entropy map. Plus markers are the same as in panel (a).

\section{b) $\mathrm{NH}_{3}$ Emission}

Maps of the integrated $\mathrm{NH}_{3}(1,1)$ and $(2,2)$ line emission are shown in Figures $4 a$ and $4 b$, respectively. The $(1,1)$ main line emission arises from an elongated region, $\sim 50^{\prime \prime} \times 20^{\prime \prime}$, oriented at roughly the same position angle as the dust continuum and $\mathrm{C}^{\mathbf{1 8}} \mathrm{O}$ emission region. This morphology is shared by the $(2,2)$ emission, which measures $44^{\prime \prime} \times 20^{\prime \prime}$ in the $13^{\prime \prime}$ resolution map. The $\mathrm{NH}_{3}(1,1)$ emission region includes, but is not centered on, the $2.7 \mathrm{~mm}$ continuum source. The $\mathrm{NH}_{3}(1,1)$ peak is offset along the minor axis, $8^{\prime \prime} .5$ southeast of the $\mathrm{C}^{18} \mathrm{O}$ peak, approximately an $8 \sigma$ separation. To within the $13^{\prime \prime}$ resolution of the map, the $\mathrm{NH}_{3}(2,2)$ emission is centered on the $2.7 \mathrm{~mm}$ continuum source.

Maps of the emission in the two central $\mathrm{NH}_{3}(1,1)$ channels, which include almost all of emission from the main hyperfine components, are shown in Figure 5 . In the $(1,1)$ line, the brightest regions have fluxes of $\sim 250 \mathrm{mJy} \mathrm{beam}^{-1}$ corresponding to a brightness temperature of $16.5 \mathrm{~K}$, assuming the

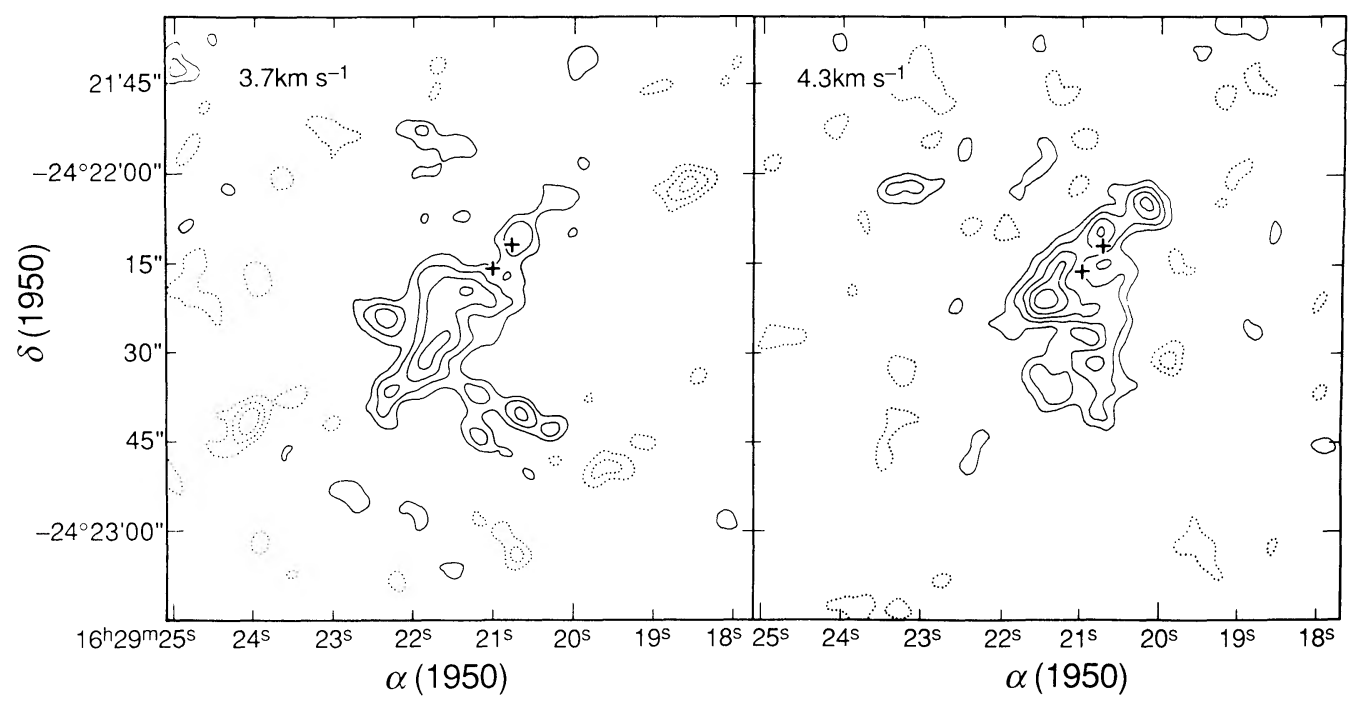

Fig. 5.-Maps of the $\mathrm{NH}_{3}(1,1)$ emission in the central two channels covering the main hyperfine component are presented. The central velocity of each channel

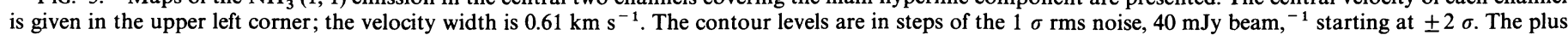
marks denote the positions of the $6 \mathrm{~cm}$ radio sources (Wootten 1989). The synthesized beam size is the same as in Fig. 4. 
$6^{\prime \prime}$ beam is filled. These bright regions appear to have a clumpy distribution which becomes accentuated in higher spatial resolution maps (an effect which is also present in the $[2,2]$ maps). There is also a general shift in the emission centroid between the $3.7 \mathrm{~km} \mathrm{~s}^{-1}$ (panel a) and $4.3 \mathrm{~km} \mathrm{~s}^{-1}$ (panel b) channels; unfortunately, the velocity resolution of the present data is insufficient to determine if this shift represents significant rotational motion. Interestingly, the total width of the $\mathrm{NH}_{3}(1,1)$ line $(\mathrm{FWZI})$ is only $1.2 \mathrm{~km} \mathrm{~s}^{-1}$, in stark contrast to the $2.4 \mathrm{~km} \mathrm{~s}^{-1}$ velocity spread evident in $\mathrm{C}^{18} \mathrm{O}$. Thus, both spatial and velocity information suggest that the $\mathrm{C}^{18} \mathrm{O} J=1-0$ and $\mathrm{NH}_{3}(1,1)$ emission lines in the interferometric data arise in significantly different regions.

The inner two satellite lines of the $\mathrm{NH}_{3}(1,1)$ line were also detected. Although the signal to noise is poor, these lines confirm the basic structure seen in Figures 4 and 5. The satellite lines indicate that the brightest regions have optical depths of $\sim 4$ in the main line, using the standard modeling for $\mathrm{NH}_{3}$ (Ho and Townes 1983).

The $\mathrm{NH}_{3}(2,2)$ emission shown in Figure $4 b$ arises from two $0.3 \mathrm{~km} \mathrm{~s}^{-1}$ channels with maximum brightness temperatures of $1.6 \mathrm{~K}$, assuming the $13^{\prime \prime}$ beam is filled. Structure is clearly present on smaller scales, as peak brightness temperatures of $4 \mathrm{~K}$ can be seen in spectra made using a $6^{\prime \prime}$ beam. The $(2,2)$ line, which is $0.6 \mathrm{~km} \mathrm{~s}^{-1}$ wide, is the narrowest of the observed lines. Data from the $43 \mathrm{~m}$ telescope do show weak wings in the $(2,2)$ line extending over the velocity range in the map of $\mathrm{C}^{18} \mathrm{O}$ emission (Wootten 1989b, unpublished data) but the wings are below the sensitivity of the interferometer data. The $(2,2)$ data reinforce the conclusion based upon the $(1,1)$ data that the majority of the $\mathrm{NH}_{3}$ emission arises in a very different region from that producing dust continuum emission.

\section{c) ${ }^{12} \mathrm{CO}$ Emission}

The ${ }^{12} \mathrm{CO}$ emission detected by the interferometer is located primarily near the region of dust continuum and $\mathrm{C}^{18} \mathrm{O}$ emission, in the velocity range from $-2.5 \mathrm{~km} \mathrm{~s}^{-1}$ to $13.1 \mathrm{~km} \mathrm{~s}^{-1}$, suggesting that it is associated with the high velocity molecular outflow found by single-dish observations (Wootten and Loren 1987; Walker et al. 1988). The main high velocity emission is segregated into two major concentrations with the blue-shifted gas $\left(-2.5\right.$ to $\left.2.7 \mathrm{~km} \mathrm{~s}^{-1}\right)$ lying roughly 5.5 east of the redshifted gas (5.3 to $\left.13.1 \mathrm{~km} \mathrm{~s}^{-1}\right)$, slightly more than a full beam offset. This polarity of the $\mathrm{CO}$ emission agrees with the larger scale distribution of high-velocity molecular gas. The centerline of the blueshifted and redshifted emission lies roughly between the $2 \mathrm{~cm}$ radio sources and within the $2.7 \mathrm{~mm}$ continuum source. The peak fluxes, in the range from 1.6 to $4.5 \mathrm{Jy}$ beam $^{-1}(2.7$ to $7.5 \mathrm{~K}$ brightness temperature), represent only a small fraction of the high-velocity emission in a single-dish spectrum. Almost all of the emission within $2.5 \mathrm{~km} \mathrm{~s}^{-1}$ of the cloud rest velocity $\left(4.0 \mathrm{~km} \mathrm{~s}^{-1}\right)$ was resolved out; the residual flux could be associated with the outflow but is more likely a result of small fluctuations in $\mathrm{CO}$ emission in the extended cloud.

Along with the emission toward IRAS 16293-2422, nine isolated clumps of high-velocity $\mathrm{CO}$ emission were detected within the lobes of the molecular outflow. These emission clumps have a velocity width of two or more channels $(\geq 5.2$ $\mathrm{km} \mathrm{s}^{-1}$ ) and range in $V_{\mathrm{LSR}}$ from -7.7 to $15.7 \mathrm{~km} \mathrm{~s}^{-1}$. Although there is no tendency for the clumps to reside in the large-scale outflow lobes of the corresponding velocity, the complex nature of the outflow and the high velocities of the emission suggest that they are associated with the outflow. Only a small fraction of the single-dish flux from the high-velocity $\mathrm{CO}$ gas is recovered by the interferometer implying that most of the gas is too smoothly distributed to be adequately sampled by the interferometer observations, sizes $>35^{\prime \prime}$.

\section{DISCUSSION}

As a framework for discussion of the current observations, we will divide the IRAS $16293-2422$ region into four components: the static core, the disk, the outer disk region, and the outflow. A schematic model for IRAS 16293-2422 and its surrounding static core is presented in Figure 6 and discussed in the following sections.

\section{a) The Static Core}

IRAS $16293-2422$ is situated deep within a high-column density core in a filament of the $\rho$ Oph cloud complex. The core was first mapped in detail by Wootten and Loren (1987) who found $\mathrm{DCO}^{+}$and $\mathrm{NH}_{3}$ emission over a $3^{\prime}$ diameter area peaking $2^{\prime}$ east of the $I R A S 16293-2422$. A $C^{18} \mathrm{O}$ strip map by Wootten and Loren (1987) also indicates that IRAS $16293-2422$ lies within a high-column density region. The narrow CS absorption lines as well as narrow components of the $\mathrm{NH}_{3}$ and $\mathrm{C}^{18} \mathrm{O}$ emission lines are ascribed to an absorbing layer (Menten et al. 1987) which we associate with the static core.

The 1.1 resolution $\mathrm{C}^{18} \mathrm{O}$ and $\mathrm{C}^{17} \mathrm{O}$ single-dish spectra presented in Figure 3 provide additional information about the core gas. Simple LVG models of the four isotropic CO lines are consistent with gas densities from $5 \times 10^{3}$ to $5 \times 10^{4} \mathrm{~cm}^{-3}$ and $\mathrm{C}^{18} \mathrm{O}$ column densities from $11-5 \times 10^{15} \mathrm{~cm}^{-2}$ for gas temperatures from $11.5 \mathrm{~K}$ to $30 \mathrm{~K}$. Spectra of the $\mathrm{NH}_{3}(1,1)$ and $(2,2)$ lines obtained with the NRAO $43 \mathrm{~m}$ (Wootten $1989 \mathrm{~b}$, unpublished observations) indicate that the rotational temperature is $15 \pm 1 \mathrm{~K}$, implying that the kinetic temperature associated with the static core is $15-20 \mathrm{~K}$. The most probable ranges for the gas density, $\mathrm{C}^{18} \mathrm{O}$ column density, and $\mathrm{H}_{2}$ column density consistent with this temperature range and our single-dish spectra are given in Table 2 . These values are only modestly different from the conditions derived from the $\mathrm{NH}_{3}$ emission by Menten et al. (1987) and Mizuno et al. (1990). Optical depth in the $\mathrm{C}^{\mathbf{1 8}} \mathrm{O} J=1-0$ transition in this gas, 0.4

TABLE 2

Conditions in the Static Core

\begin{tabular}{lcc}
\hline \multicolumn{1}{c}{ Property } & Value & Method \\
\hline Temperature $(\mathrm{K}) \ldots \ldots \ldots \ldots$ & $15-20$ & $\mathrm{NH}_{3}$ excitation \\
Density $\left(\mathrm{cm}^{-3}\right) \ldots \ldots \ldots \ldots \ldots$ & $1-2 \times 10^{4}$ & $\mathrm{C}^{18} \mathrm{O}, \mathrm{C}^{17} \mathrm{O}$ excitation \\
$\tau\left(\mathrm{C}^{18} \mathrm{O} J=1-0\right) \ldots \ldots \ldots \ldots$ & $\mathrm{C}^{18} \mathrm{O}$ excitation \\
$N\left(\mathrm{C}^{18} \mathrm{O}\right)\left(\mathrm{cm}^{-2}\right) \ldots \ldots \ldots \ldots \ldots$ & $6-7.5 \times 10^{15}$ & $\mathrm{C}^{18} \mathrm{O}$ excitation \\
$N\left(\mathrm{H}_{2}\right)\left(\mathrm{cm}^{-2}\right) \ldots \ldots \ldots \ldots \ldots$ & $3-4 \times 10^{22}$ & assumes $\mathrm{C}^{18} \mathrm{O} / \mathrm{H}_{2}=2 \times 10^{-7}$ \\
$A_{v}(\mathrm{mag}) \ldots \ldots \ldots \ldots \ldots \ldots$ & $33-44$ & assumes $A_{v}=1.8 \times 10^{21} N\left(\mathrm{H}_{2}\right) \mathrm{cm}^{-2} \mathrm{mag}^{-1}$ \\
\hline
\end{tabular}




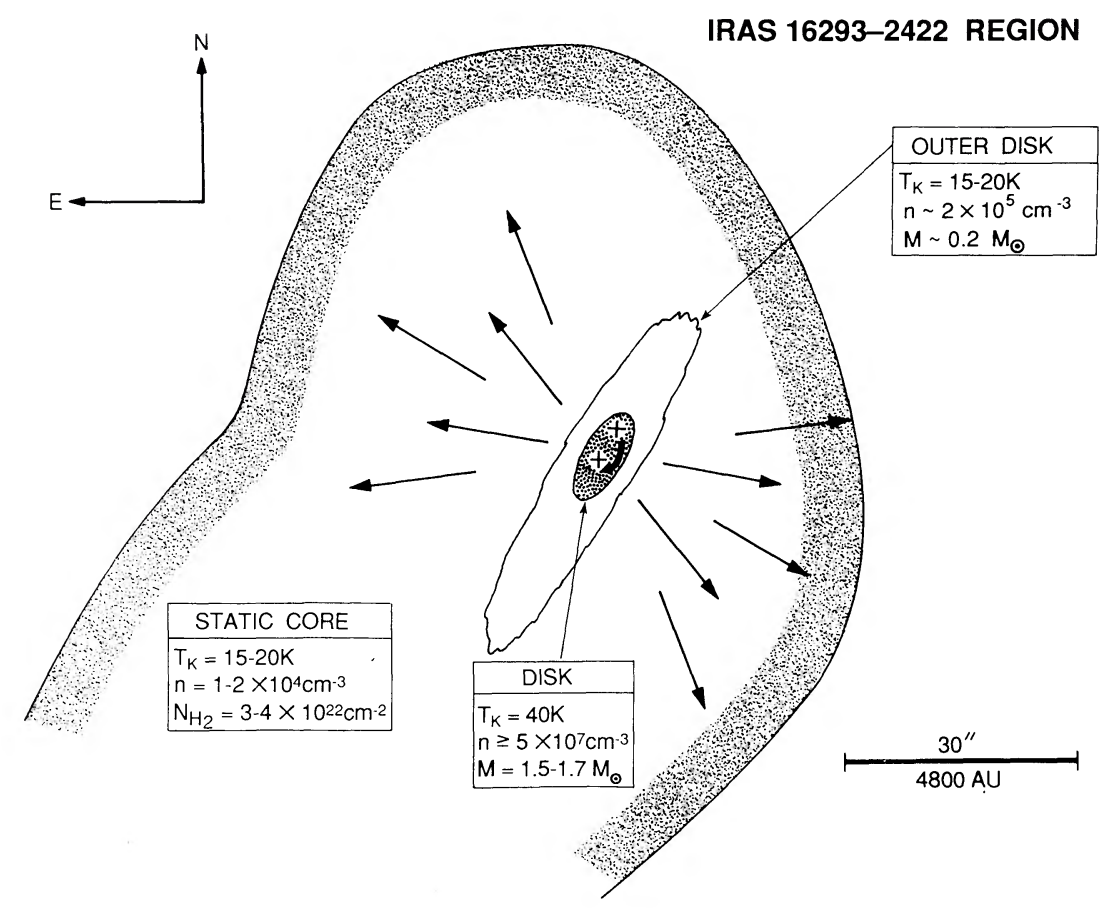

FIG. 6.-A schematic map of IRAS 16293-2422 and surrounding region is sketched above. The four major components discussed in $\S$ IV are shown and

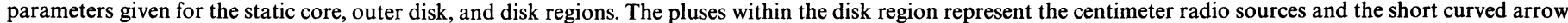

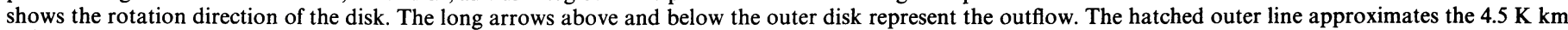
$\mathrm{s}^{-1}$ contour of the integrated $\mathrm{C}^{18} \mathrm{O} J=1-0$ emission $\left(T_{R}^{*}\right)$ from the static core (Walker and Wilking 1989).

0.7 , is sufficient to absorb $30 \%-50 \%$ of the low-velocity line emission from the disk and outer disk region. Since the OVRO observations resolve out emission from the static core, the end result is a decrease in observed $\mathrm{C}^{18} \mathrm{O}$ line flux near line center. Indeed, high-spatial resolution profiles from the IRAM $30 \mathrm{~m}$ (Menten et al. 1987) and NRO $45 \mathrm{~m}$ (Mizuno et al. 1990) telescopes show no intensity decrease at line center as the static core emission fills in the absorption.

\section{b) The Disk}

The warm $(\sim 40 \mathrm{~K})$, high-column density gas and dust region responsible for the bulk of the infrared and millimeter emission and the compact $\mathrm{C}^{18} \mathrm{O}$ line emission is identified as the disk component. It covers $\sim 12$ ", $2000 \mathrm{AU}$, in the longest dimension and oriented roughly orthogonal to the $\mathrm{CO}$ outflow and the local magnetic field (Wootten and Loren 1987). The velocity-position diagram, Figure 2, indicates rotational motion within this region but no central object has been detected at any wavelength. In fact, $2 \mathrm{~cm}$ and $6 \mathrm{~cm}$ VLA maps (Wootten 1989a) suggest the presence of two independent ionization sources within the disk component. Thus, it is not clear if it is correct to view this material as a circumstellar disk, a circumbinary disk, or as a combination of two disks in a binary star system. For the present discussion, we will view the material as a single coherent structure, either circumstellar or circumbinary, since our observations contain little information about structure on the scale of the centimeter radio sources and $\mathrm{H}_{2} \mathrm{O}$ masers.

The mass of the disk region can be estimated using three independent methods based on velocity information, $2.7 \mathrm{~mm}$ continuum emission, or total $\mathrm{C}^{\mathbf{1 8}} \mathrm{O}$ emission. The total mass of the disk region, including any young stellar objects, can be estimated from the velocity extremes at the ends of the emission region. The full velocity shift at $\pm 6^{\prime \prime}$ from the disk cen- troid covers $2.4 \mathrm{~km} \mathrm{~s}^{-1}$. Assuming the gas is in circular orbit, the included mass is $1.5 M_{\odot} \sin i^{-1}$. The current observations limit the inclination angle to $\geq 60^{\circ}$, assuming an intrinsically circular disk, implying that the mass is $1.5-1.7 M_{\odot}$.

The total $2.7 \mathrm{~mm}$ continuum emission gives an estimate of the mass of gas and dust in the disk component. This calculation was done by Mundy, Wilking, and Myers (1986), assuming $N\left(\mathrm{H}_{2}\right) / \tau_{\text {dust }}=6 \times 10^{24} \mathrm{~cm}^{-2}$ (Hildebrand 1983), yielding masses of $0.9,2.3$, and $6.0 M_{\odot}$ for $\lambda^{-1}, \lambda^{-1.5}$, and $\lambda^{-2}$ emissivity laws, respectively.

Lastly, the $\mathrm{C}^{\mathbf{1 8}} \mathrm{O}$ emission can be used to estimate a gas mass. Assuming the molecule is thermalized and the $J=1-0$ transition optically thin, the total $\mathrm{C}^{18} \mathrm{O} J=1-0$ emission, integrated over the source and velocity $(F)$, is related to the gas temperature $(T)$, the distance to the object $(D)$, and the total number of $\mathrm{C}^{18} \mathrm{O}$ molecules $\left(N\left[\mathrm{C}^{18} \mathrm{O}\right]\right)$.

$$
F=\frac{h c}{4 \pi} f_{0}(T) B_{0,1}\left(1-e^{-h v / k T}\right) B_{v}(T) N\left(\mathrm{C}^{18} \mathrm{O}\right) D^{-2},
$$

where $f_{0}(T)$ is the fractional population in the $J=0$ level, $B_{0,1}$ is the Einstein absorption coefficient for the $J=0$ level, and $B_{v}(T)$ is the Planck function. The total number of $\mathrm{C}^{18} \mathrm{O}$ molecules is related to the gas mass $(M)$ by the fractional abundance of $\mathrm{C}^{18} \mathrm{O}, X\left(\mathrm{C}^{18} \mathrm{O}\right)=N\left(\mathrm{C}^{18} \mathrm{O}\right) /\left(M / \mu m_{\mathrm{H}}\right)$, yielding

$$
F=\frac{h c}{4 \pi} f_{0}(T) B_{0,1}\left(1-e^{-h v / k T}\right) B_{v}(T) \frac{M}{\mu m_{\mathrm{H}}} X\left(\mathrm{C}^{18} \mathrm{O}\right) D^{-2},
$$

or evaluated in convenient units,

$$
F\left(\mathrm{Jy} \mathrm{km} \mathrm{s}^{-1}\right)=53.8\left(\frac{M}{1 M_{\odot}}\right)\left(\frac{150}{D_{\mathrm{pc}}}\right)^{2}\left(\frac{X\left(\mathrm{C}^{18} \mathrm{O}\right)}{2 \times 10^{-7}}\right) .
$$

The total $\mathrm{C}^{18} \mathrm{O}$ flux in Figure 1 is $12 \mathrm{Jy} \mathrm{km} \mathrm{s}^{-1}$ corresponding 
to $0.22 M_{\odot}$. This value is uncertain due to the large uncertainty in $X\left(\mathrm{C}^{18} \mathrm{O}\right)$ and likely underestimates the true mass due to absorption near line center by the static core gas and the possibility that the $\mathrm{C}^{\mathbf{1 8}} \mathrm{O}$ line becomes optically thick in some regions.

While the estimate using the gas dynamics, yields the most reliable measure of the total mass within a $1000 \mathrm{AU}$ radius, the current velocity information does not permit the decomposition of this 1.5-1.7 $M_{\odot}$ into contributions from gas and embedded young object(s). The $\mathrm{C}^{18} \mathrm{O}$ and continuum emission mass estimates measure the disk mass alone but are subject to large uncertainties with the $\mathrm{C}^{\mathbf{1 8}} \mathrm{O}$ mass estimate suffering from the additional problem that $30 \%-50 \%$ of the disk emission close to line center is absorbed by the static core gas. Higher resolution molecular line observations of the region will be necessary to determine its rotation curve and derive the mass distribution within the disk. If we assume that $1 M_{\odot}$ of gas and dust is in the disk, a lower limit to the gas density is $5 \times 10^{7}$ $\mathrm{cm}^{-3}$, where the unresolved minor axis was assumed to be $6 "$.

\section{c) Ammonia and the Outer Disk Region}

The morphology of the VLA $\mathrm{NH}_{3}$ maps strongly suggests that IRAS 16293-2422's influence extends well beyond the 1000 AU radius of the disk. The ammonia emission arises from an elongated region which extends $8000 \mathrm{AU}$ in diameter at a position angle similar to that of the disk. Both the maximum $\mathrm{NH}_{3}(1,1)$ brightness temperature of $16.5 \mathrm{~K}$ and the kinetic temperature estimate of $13 \pm 2 \mathrm{~K}$, derived from a comparison of our $(2,2)$ data with the $(1,1)$ data smoothed to a resolution of $13^{\prime \prime}$, suggest a gas temperature comparable to that of the static core. Using the $\mathrm{NH}_{3}$ excitation calculations by Stutzki and Winnewisser $(1985)$, assuming $\tau(1,1)=3.5 \pm 1.5$ and $T_{k}=$ $15 \pm 2 \mathrm{~K}$, the average spatial density in the outer disk region is $(1.5 \pm 0.5) \times 10^{5} \mathrm{~cm}^{-3}$ and the column density $N\left(\mathrm{NH}_{3}\right) \sim$ $(2 \pm 0.5) \times 10^{15} \mathrm{~cm}^{-2}$. Assuming a symmetric disk geometry with a disk thickness of $20^{\prime \prime}$, the mass of material in the $\mathrm{NH}_{3}$ emission region is $\sim 0.2 M_{\odot}$ yielding an $\mathrm{NH}_{3}$ fractional abundance of $\sim 2 \times 10^{-7}$. These results for density and mass are significantly model dependent and could easily suffer systematic uncertainties of a factor of 2-3.

The most surprising aspect of the $\mathrm{NH}_{3}$ data is weakness of emission from the more massive inner disk component. If $\mathrm{NH}_{3}$ were present in the disk component with a normal interstellar abundance, $10^{-7}$ (Ho and Townes 1983), the ammonia column density would be $\sim 10^{17} \mathrm{~cm}^{-2}$ implying $\tau \gg 1$ in the $(1,1)$ and $(2,2)$ transitions and expected brightness temperatures of $40 \mathrm{~K}$, equal to the kinetic temperature. This is more than twice the brightness temperature observed anywhere in the $(1,1)$ map and far stronger than any emission in the $(2,2)$ map. The narrow $\mathrm{NH}_{3}$ linewidth relative to $\mathrm{C}^{\mathbf{1 8}} \mathrm{O}$ and the low $\mathrm{NH}_{3}$ rotation temperature confirm this lack of emission from the disk gas. Since the weak $(2,2)$ line rules out the presence of high temperatures $\left(T_{\mathrm{K}} \gg 40 \mathrm{~K}\right)$ in the inner disk component, the dearth of ammonia emission represents a deficiency in ammonia abundance in the inner disk. The cause of this deficiency remains undetermined, but possible causes include depletion onto grains or changes in the chemistry. At first thought depletion seems unattractive in a region which is warmer than other portions of the cloud. However, depletion may have occurred in an earlier colder phase in the history of IRAS 16293-2422 and the chemistry may not have maintained equilibrium as the gas and dust are heated by the forming star. Such a scenario has been suggested for the much warmer gas near IRc 2 in the Orion molecular cloud (Walmsley et al. 1987).

\section{d) The Outflow}

The ${ }^{12} \mathrm{CO}$ interferometer results show that a molecular outflow originates from the high-column density inner disk. There is insufficient resolution to associate this gas with either of the compact $2 \mathrm{~cm}$ sources within the disk component; neither is there evidence on this small scale for the quadrupolar nature of the large-scale outflow (e.g., Walker et al. 1988). Additional high-velocity emission was detected away from the central source but this emission poorly traces the large-scale structure of the outflow. Several other outflow regions mapped with the OVRO array by Scoville et al. (1985) yield similar results with the base of the outflow evident but lobes resolved out. Apparently, the region of the IRAS 16293-2422 outflow covered by our observations has little structure on spatial scales from $6^{\prime \prime}$ to $30^{\prime \prime}$. With the interferometer recovering such a small fraction of the total $\mathrm{CO}$ flux in the outflow, physical interpretation of the observed flux is difficult. For example, the clumps of high-velocity emission away from the disk region do not appear to correspond to neither edges of the outflow nor the clumps within the outflow identified by Walker et al. (1988).

\section{SUMMARY}

Interferometric $\mathrm{C}^{\mathbf{1 8}} \mathrm{O}$ and $\mathrm{NH}_{3}$ observations have begun to reveal the dynamics and morphology of the gas around the young far-infrared source IRAS 16293-2422. The $\mathrm{C}^{18} \mathrm{O}$ observations, with $6.3 \times 4$ ".5 resolution $(1000 \times 720$ AU), detect emission from an elongated region, approximately 2000 $\mathrm{AU}$ in the longest dimension. This region is nearly identical in extent to the $40 \mathrm{~K}$ dust structure previously observed in the 2.7 $\mathrm{mm}$ continuum. The $\mathrm{C}^{18} \mathrm{O}$ emission region exhibits a velocity difference of $2.4 \mathrm{~km} \mathrm{~s}^{-1}$ from end-to-end. We interpret this structure as a rotating disk surrounding the young star or stars responsible for heating the gas and dust. From the dynamics of the gas, we estimate that the included mass of IRAS $16293-2422$ (disk plus embedded object[s]) is $1.5-1.7 M_{\odot}$.

At 6" (960 AU) resolution, the majority of the $\mathrm{NH}_{3}$ emission arises from a $50^{\prime \prime}$ by $20^{\prime \prime}$ region surrounding and aligned with the $\mathrm{C}^{18} \mathrm{O}$ emission. The $\mathrm{NH}_{3}$ line is much narrower than the $\mathrm{C}^{18} \mathrm{O}$ line. The gas traced by the $\mathrm{NH}_{3}$ emission is characterized by $T_{\mathrm{K}}=15-20 \mathrm{~K}, n\left(\mathrm{H}_{2}\right)=1-2 \times 10^{5} \mathrm{~cm}^{-3}$, and a total mass of $\sim 0.2 M_{\odot}$, all of which are very different from the conditions in the inner "disk" region. It appears that the $\mathrm{NH}_{3}$ emission mainly arises from a ring 3000-4000 AU in radius; the dearth of emission inside this ring, and especially from the $2.7 \mathrm{~mm}$ continuum source, is likely to be due to a large decrease in $\mathrm{NH}_{3}$ abundance rather than unfavorable excitation conditions. With a gas density approximately one order of magnitude higher than the surrounding static core, the $\mathrm{NH}_{3}$ emitting region may constitute a diffuse outer disk formed during the creation of the central source(s).

Our interferometric $\mathrm{CO}$ observations resolve out most of the high-velocity gas seen in single-dish observations. However, concentrations of both redshifted $\left(5.3\right.$ to $\left.13.1 \mathrm{~km} \mathrm{~s}^{-1}\right)$ and blueshifted $\left(-2.5\right.$ to $\left.2.7 \mathrm{~km} \mathrm{~s}^{-1}\right)$ gas were detected associated with the inner disk region. The separation and orientation of this emission suggests that it is delineating the base of the bipolar outflow.

We thank J. Blackwell and J. Howe for obtaining the $\mathrm{C}^{17} \mathrm{O}$ 
and $\mathrm{C}^{18} \mathrm{O} J=2-1$ spectra, J. Mangum for help in obtaining the VLA data, and the Owens Valley staff for their efforts in maintaining and improving the millimeter interferometer. The
SIS junctions used in the receivers at Owens Valley were provided by R. E. Miller of A.T.T.B.L. L. G. M. acknowledges support from NSF grant AST87-14405.

\section{REFERENCES}

Hildebrand, R. H. 1983, Ouart. J.R.A.S., 24, 267.

Ho, P. T. P., and Townes, C. 1983, Ann. Rev. Astr. Ap., 21, 239

Menten, K. M., Serabyn, E., Gusten, R., and Wilson, T. L. 1987, Astr. Ap. (Lets), 177, L57.

Mizuno, A., et al. 1990, Ap. J., submitted.

Mundy, L. G., Wilking, B. A., and Myers, S. T. 1986, Ap. J. (Letters), 311, L75.

Scoville, N. Z., Sargent, A. I., Sanders, D. B., Claussen, M. C., Masson, C. R., Lo, K. Y., and Phillips, T. G. 1985, Ap. J., 303, 416.

Stutzki, J., and Winnewisser, G. 1985, Astr. Ap., 148, 254.

Walker, C. K., Lada, C. J., Young, E. T., Maloney, P. R., and Wilking, B. A. 1986, Ap. J.(Letters), 309, L47.

Walker, C. K., Lada, C. J., Young, E. T., and Margulis, M. 1989, Ap. J., 332, 335.

Walker, C. K., and Wilking, B. A. 1989, unpublished data.

Walmsley, M., Hermsen, W., Henkel, C., Mauersberger, R., and Wilson, T. L. 1987, Astr. Ap., 172, 311.

Wilking, B. A., and Claussen, M. J. 1987, Ap. J. (Letters), 320, L133.

Wootten, H. A. $1989 a$, Ap. J., 337, 858.

.1989b, unpublished data.

Wootten, H. A., and Loren, R. B. 1987, Ap. J., 317, 220.

LeE G. MUNDY: Astronomy Program, University of Maryland, College Park, MD 20742

Bruce A. Wilking: Department of Physics, University of Missouri-St. Louis, 8001 Natural Bridge Road, St. Louis, MO 63121

AlWyn WootTEn: National Radio Astronomy Observatory, Edgemont Road, Charlottesville, VA 22903-2475 